\title{
Relation between fentanyl dose and level of consciousness measured with patient state index during spinal anesthesia for elective cesarean section
}

$\underline{\text { Iwata H. }}^{1}$, Mimuro S. ${ }^{2}$, Sakai H. ${ }^{1}$, Takahashi K. ${ }^{1}$, Shiraishi Y. ${ }^{1}$, Nakajima Y. ${ }^{2}$

${ }^{1}$ Fujieda Municipal General Hospital, Dept of Anaesthesiology, Fujieda, Japan,

${ }^{2}$ Hamamatsu University School of Medicine, Dept of Anaesthesiology \& Intensive Care, Hamamatsu, Japan

\section{Introduction}

- In spinal anesthesia for cesarean section, the addition of fentanyl to the local anesthetic has been reported to improve perioperative analgesia. However, there is only limited knowledge on sedative effects of the added fentanyl.

- We evaluated the relationship between fentanyl dose and the level of consciousness, as measured by a patient state index (PSI) during spinal anesthesia for cesarean section.

\section{Subjects and Methods}

- Subjects: 15 patients scheduled for elective cesarean section under combined spinal-epidural anesthesia.

- Patients were divided into 3 groups according to fentanyl dose (10, 15 , or $20 \mu \mathrm{g}$ ). Each group comprised 5 patients.

Exclusion criteria

- History of neurological disorders

- Block level after spinal anesthesia was below the Th4

- Analgesics were required during the operation

\section{Anesthesia}

Epidural : A catheter was placed at the Th12/L1. $1 \%$ lidocaine $(3 \mathrm{ml})$ was administered. No additional dose was administered.

Spinal : High-density bupivacaine $(2.2 \mathrm{~mL})+$ fentanyl $(10$, 15 , or $20 \mu \mathrm{g}$ ) was administered at the $\mathrm{L} 3 / 4$.

- After administration, oxygen (5 L) was administered using a mask, and the PSI was measured using a SedLine ${ }^{\circledR}$ monitor (Masimo Corp., Irvine CA).

- The PSI value, respiratory rate and $\mathrm{SpO} 2$ during the operation were recorded every 2 minutes.

- The period between child delivery and completion of the surgery, the proportions of time with PSI values $<90$ and 80 , respectively, were calculated.

Statistical analysis

One-way analysis of variance (ANOVA) followed by Scheffe's test for intergroup comparisons. $\mathrm{P}<0.05$ was regarded as significant.

\section{Results}

- All patients were included in 3 groups without exclusion.

- No patient showed an $\mathrm{SpO} 2<95 \%$ or respiratory rate $<8 / \mathrm{min}$.

- No significant difference was observed in the height, body weight, age, gestational age or the surgical time among the 3 groups.

$(\%)$

100

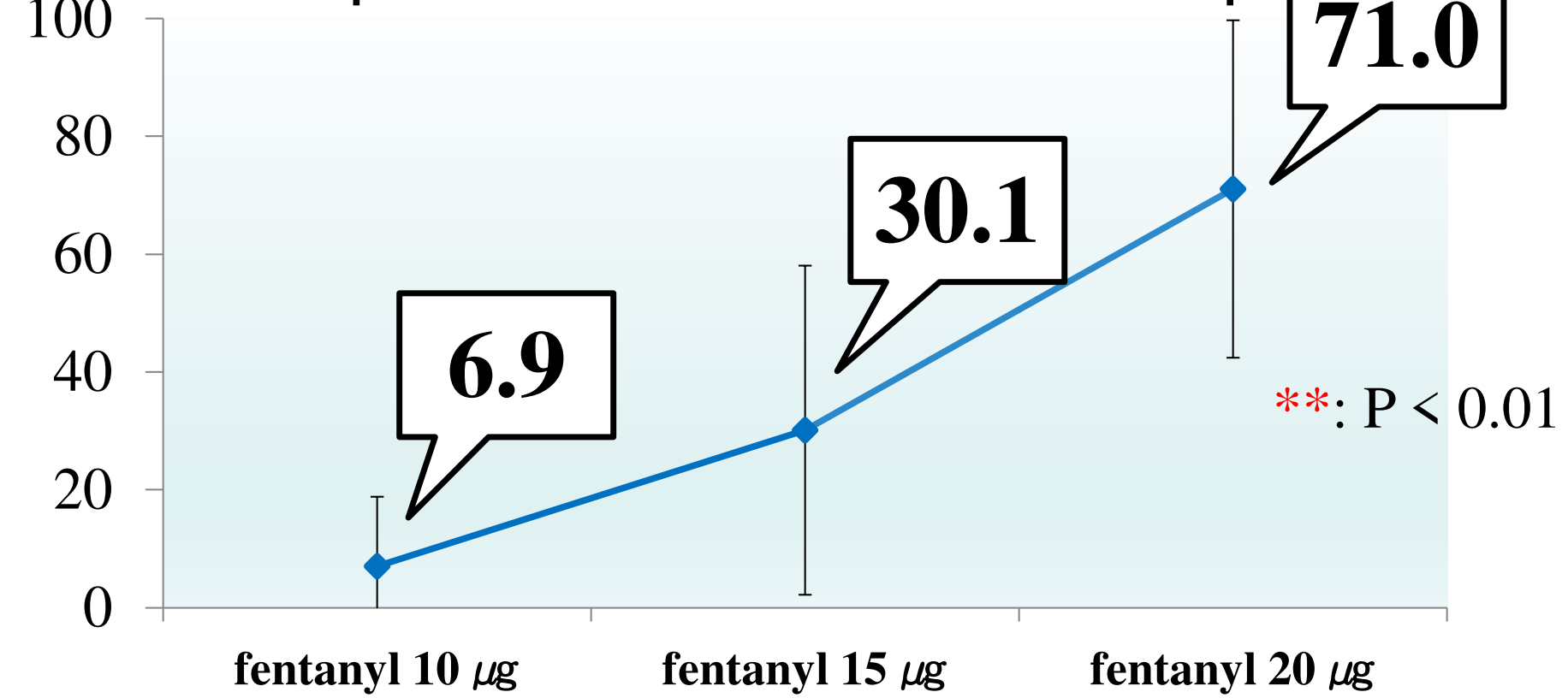

\begin{tabular}{ccccc}
\hline & $10 \mu \mathrm{g}$ & $15 \mu \mathrm{g}$ & $20 \mu \mathrm{g}$ & P-value \\
\hline Height $(\mathrm{cm})$ & $156.6 \pm 2.5$ & $155.8 \pm 2.0$ & $158.5 \pm 2.4$ & $\mathrm{NS}$ \\
Weight $(\mathrm{kg})$ & $56.7 \pm 2.8$ & $55.1 \pm 4.0$ & $72.6 \pm 6.0$ & $\mathrm{NS}$ \\
Age (yr) & $30.6 \pm 2.6$ & $30.8 \pm 1.7$ & $32.8 \pm 1.8$ & $\mathrm{NS}$ \\
Gestational age (wk) & $37.7 \pm 0.4$ & $38.3 \pm 0.3$ & $38.9 \pm 0.4$ & $\mathrm{NS}$ \\
Surgical time (min) & $58.4 \pm 6.3$ & $56.6 \pm 2.3$ & $63.2 \pm 6.2$ & $\mathrm{NS}$ \\
\hline
\end{tabular}

Figure 1: Patients' characteristics : mean \pm SD
(\%)

\section{PSI $<80$}

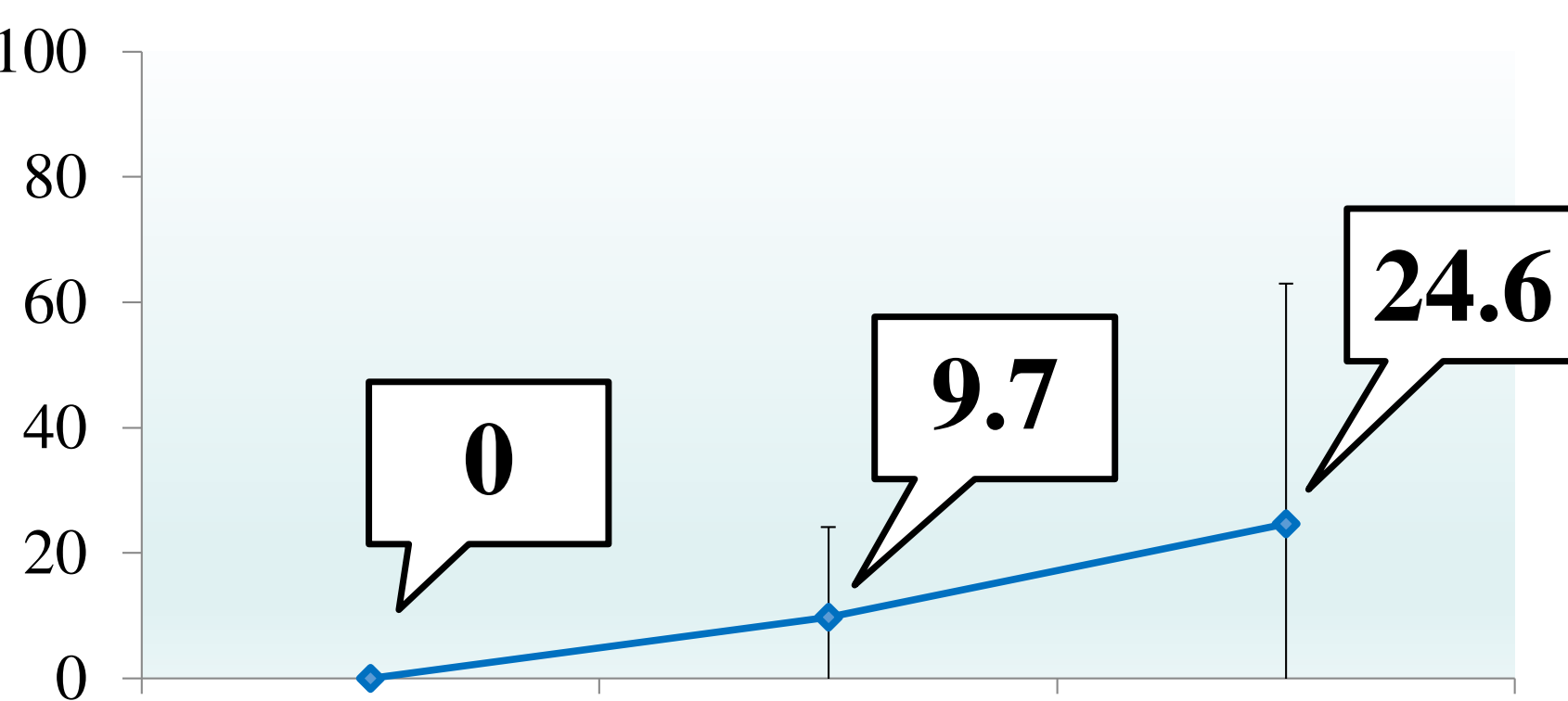

fentanyl $10 \mu \mathrm{g} \quad$ fentanyl $15 \mu \mathrm{g}$

fentanyl $20 \mu \mathrm{g}$

Figure 2: The proportion of time with PSI values $<90$ and 80 between child delivery and operative completion: mean \pm SD

- PSI values $<90$ : There was a significant difference between fentanyl dose of 10 and $20 \mu \mathrm{g}$.

- PSI values $<80$ : There were no significant differences.

\section{Discussion}

- The results of the present study demonstrate that PSI values decrease in dose-dependent manner with increasing dose of fentanyl.

- PSI is a clinically validated measure of the effect of anaesthesia and sedation. PSI values $<50$ are associated with a low probability of consciousness. Drover assessed the relationship between PSI values and observer's assessment of alertness/sedation (OAA/S) scale scores and reported a positive correlation. PSI values changed even during mild sedation (OAA/S, 5-4). When OAA/S scale score was 4, PSI values were approximately 80.

- Schneider et al. reported PSI values of awake state were 98-99. In the present study, fentanyl dose $\geq 15 \mu \mathrm{g}$ tended to increase the proportion of time with PSI values $<90$. This result may indicate that administration of fentanyl at doses $\geq 15 \mu \mathrm{g}$ has mild sedative effect.
Limitations $\cdot$ A small number of patients
OAA/S scale score 5: Responds readily to name spoken in normal tone
- OAA/S scale scores were not evaluated
4: Lethargic response to name spoken in normal tone

\section{Conclusion}

- PSI values decrease in a dose-dependent manner with increased dose of fentanyl added to local anesthetic during spinal anesthesia for cesarean section. PSI values were more likely to be $<90$ when fentanyl was administered at doses $\geq 15 \mu \mathrm{g}$. 